\title{
On the Perturbation of Critical Values of Maximum-Minimum
} Type

\author{
F. BENKERT
}

The stability of critical values of a functional under small perturbations is investigated. There are considered critical values of maximum-minimum type of functionals which are related to boundary value and eigenvalue problems for semilinear elliptic partial differential equations.

Key words: critical values of mazimum-minimum type, semilinear elliptic partial differential equations AMS subject classification: $58 \mathrm{E} 05,35 \mathrm{~J} 20,58 \mathrm{C} 40$

\section{Introduction}

In this paper we will deal with critical values of maximum-minimum type of functionals which arise in the study of boundary value and eigenvalue problems for semilinear elliptic partial differential equations. Our aim is to investigate such critical values under perturbations of the primary functional. In order to explain the basic ideas let $X$ be a real Banach space and let $\Phi: X \rightarrow \mathbf{R}$ be a functional having the Fréchet derivative $\Phi^{\prime}$. By a critical point of $\Phi$ we mean an $u \in X$ such that $\Phi^{\prime}(u)=0$; the corresponding value $c=\Phi(u)$ is called a critical value of $\Phi$. The number

$$
c=\sup _{K \in \mathcal{K}} \inf _{u \in K} \Phi(u)
$$

where $\mathcal{K}$ is a suitable class of subsets of $X$, under certain assumptions is a criticai value of $\Phi$. Analogously one investigates critical values of the restriction of a functional $\Phi$ to a certain subset $M$ of $X$. We will study perturbation problems of the following kind:

Let $c$, defined acconding to (1), be a critical value of $\Phi$. Given $\epsilon>0$, is there a critical value $c_{*}$ of the functional $\Phi_{*}=\Phi+\Psi$ such that $c_{*} \in(c-\epsilon, c+\epsilon)$, provided the functional $\Psi$ is, in a certain sense, sufficiently small?

We will study this perturbation problem for free local extrema (minima, maxima, critical values of mountain pass type) and for critical values of the restriction of a functional to a Banach manifold $M$ in $X$ using Ekeland's variational principle and the so-called deformation theorem, respectively. The use of these techniques is essentially based on the Palais-Smale condition (PS) or on the local Palais-Smale condition (PS) $)_{c}$ at level $c$ (cf. Subsection 2.2). Because of this, the following stability problem for the Palais-Smale condition will play an important role in our 
investigations:

Let $\Phi$ satisfy the Palais-Smale condition (PS) (resp. (PS) $)_{c}$ ). Does the functional $\Phi_{*}=$ $\Phi+\Psi$ satisfy the Palais-Smale condition (PS) (resp. (PS) $c_{*}$ for all $c_{*} \in(c-\epsilon, c+\epsilon)$ ) if the functional $\Psi$ is, in a certain sense, sufficiently small?

Perturbation problems of the above kind have been treated in $[4,8]$. There are studied critical values of the restriction of $\Phi$ to a subset $M$ which is bounded and homeomorphic to the unit sphere by the radial projection mapping (for other perturbation results for critical values of functionals we refer to $[1,5-7])$.

In the present paper we are able to treat a wider class of problems, since we study critical values of the restriction of $\Phi$ to a Banach manifold $M$ in $X$ not necessarily homeomorphic to the unit sphere in $X$. This is done in Section 2, especially in Subsection 2.4, which is our main result in the application to semilinear elliptic partial differential equations. Furthermore, the investigations of the examples in Subsection 1.2 and 1.4 with respect to the perturbation problem is new.

In Section 1 we investigate free local extrema, especially minima, maxima, and critical values of mountain pass type. In Section 2 we consider critical values under smooth side conditions. The theorems in Section 1 and 2 aim to the application to boundary value and eigenvalue problems for semilinear elliptic partial differential equations. Equally, all examples are concerned with such problems. Subsection 2.5, including the Example to Theorem 4, is based on [4].

Acknowledgements. I would like to thank Prof. E. Zeidler for bringing to my attention the problem and Prof. D. Klatte for helpful suggestions in improving this paper. Furthermore I thank F. Schuricht for helpful discussions.

\section{Notation}

For a Banach space $X$ we denote the dual space by $X^{\prime}$ and the value of $f \in X^{\prime}$ at $u \in X$ by $\langle f, u\rangle$. Strong convergence (resp. weak convergence) in $X$ will be denoted by $\rightarrow$ (resp. $\rightarrow$ ). $\mathbf{R}$ (resp. $\mathbf{N}$ ) denotes the set of all real numbers (resp. positive integers). If $a, b \in \mathbf{R}$, then $(a, b)=\{x \in \mathbf{R} \mid a<x<b\}$ and $(a, b]=\{x \in \mathbf{R} \mid a<x \leq b\}$.

Let $\Phi: X \rightarrow \mathbf{R}$ be a functional on $X$. Then, $\Phi \in C^{1}(X, \mathbf{R})$ means that $\Phi$ is continuously Fréchet differentiable; $\Phi^{\prime}$ denotes its derivative. $\Phi$ is said to be weakly continuous if $u_{m} \rightarrow u$ implies $\Phi\left(u_{m}\right) \rightarrow \Phi(u)$ as $m \rightarrow \infty$.

Suppose $A: X \rightarrow X^{\prime}$ is an operator from $X$ into $X^{\prime}$. Then $A$ is said to be compact if it is continuous and maps bounded sets into relatively compact sets. The operator $A$ is said to be strongly continuous if $u_{m} \rightarrow u$ in $X$ implies $A\left(u_{m}\right) \rightarrow A(u)$ in $X^{\prime}$ as $m \rightarrow \infty$. Furthermore, 
$A$ is said to be bounded if it maps bounded sets into bounded sets. The operator $A$ is said to satisfy $(\mathrm{S})_{1}$ if $u_{m} \rightarrow u$ and $A\left(u_{m}\right) \rightarrow v$ imply $u_{m} \rightarrow u$ as $m \rightarrow \infty$. If $\Phi \in C^{1}(X, \mathbf{R})$, then the Fréchet derivative $\Phi^{\prime}$ is an operator from $X$ into $X^{\prime}$; if $\Phi^{\prime}$ is strongly continuous, then $\Phi$ is weakly continuous.

For topological spaces $M, N$ we denote by $C(M, N)$ the set of all continuous mappings of $M$ into $N$. Let $\Omega$ be a domain in $\mathbf{R}^{n}$. Then $\partial \Omega$ (resp. $\bar{\Omega}$ ) denotes its boundary (resp. its closure). $C_{b}(\bar{\Omega} \times \mathbf{R}, \mathbf{R})$ is the set of all functions from $C(\bar{\Omega} \times \mathbf{R}, \mathbf{R})$, which are uniformly bounded on $\bar{\Omega} \times \mathbf{R}$.

$L_{r}(\Omega)$ is the Lebesgue space of all $r$-integrable functions over $\Omega$ with the norm $\|\cdot\|_{r}$. Finally, in all estimates we denote by $C$ constants without prescribed value and by $C_{1}, C_{2}, \ldots$ certain values of a constant.

\section{Free local extrema}

In this section we consider functionals $\Phi \in C^{1}(X, \mathbf{R})$, where $X$ is a real Banach space. We use the notation

$$
\operatorname{crit}_{X, c} \Phi=\left\{u \in X \mid \Phi^{\prime}(u)=0, \Phi(u)=c\right\}, c \in \mathbf{R} .
$$

$\Phi$ is said to satisfy the Palais-Smale condition if the following holds:

(PS) Each sequence $\left(u_{m}\right)$ in $X$ such that $\Phi\left(u_{m}\right)$ is bounded and $\Phi^{\prime}\left(u_{m}\right) \rightarrow 0$ as $m \rightarrow \infty$ has a convergent subsequence.

\subsection{Minima and maxima}

We suppose the following assumptions:

(A1) $X$ is a real Banach space, $\Phi \in C^{1}(X, \mathbf{R})$.

(A2) $\Phi$ is bounded below on $X ; c=\inf _{u \in X} \Phi(u)$.

The following result is an immediate consequence of Ekeland's variational principle. For the proof we refer to [3].

Proposition 1: Suppose (A1), (A2) hold. Then for each $\sigma>0$ there exists $u_{\sigma} \in X$ such that $\Phi\left(u_{\sigma}\right) \leq c+\sigma$ and $\left\|\Phi^{\prime}\left(u_{\sigma}\right)\right\| \leq \sigma$.

We have the following perturbation result.

Theorem 1: Suppose (A1), (A2) hold. Let $S$ be a class of functionals $\Psi \in C^{1}(X, \mathbf{R})$ such that $\Phi_{*}=\Phi+\Psi$ satisfies (PS) for all $\Psi \in \mathcal{S}$. If $\Psi \in \mathcal{S}$ satisfies $|\Psi(u)| \leq \epsilon$ for all $u \in X$ with $\epsilon \geq 0$ and $c_{*}=\inf _{u \in X} \Phi_{*}(u)$, then $c_{*} \in[c-\epsilon, c+\epsilon]$ and crit $x_{,} c_{*} \Phi_{*} \neq 0$. 
Proof: The estimate $c_{*} \in[c-\epsilon, c+\epsilon]$ is obvious. It remains to show that crit $X_{,}, \Phi_{*} \neq 0$. The functional $\Phi$. satisfies the assumptions of Proposition 1. Hence there is a sequence $\left(u_{m}\right) \subset X$ such that $\Phi_{*}\left(u_{m}\right) \leq c_{*}+1 / m$ and $\left\|\Phi_{*}^{\prime}\left(u_{m}\right)\right\| \leq 1 / m$ for all $m \in N$. We can choose a subsequence which converges to $v \in X$, since $\Phi_{*}$ satisfies (PS). The continuity of $\Phi_{*}$ and $\Phi^{\prime}$ * yields $\Phi_{*}(v)=c_{*}$ and $\Phi^{\prime}{ }_{*}(v)=0$, i. e. , $v \in$ crit $X_{,}, \Phi_{*}$.

Remark: If $\Phi$ additionally satisfies (PS), then crit $x_{, c} \Phi \neq 0$. To investigate the stability of the critical value $c$ we have to find an appropriate class $\mathcal{S}$ which guarantees the stability of (PS) (i. e. , such that $\Phi_{*}=\Phi+\Psi$ satisfies (PS) for $\Psi \in S$ ).

Corollary 1: An analogous theorem holds for $c_{*}=\sup _{u \in X} \Phi_{*}(u)$ if $\Phi$ is bounded above on $X$.

Now, we give an example for the application of Theorem 1 to semilinear elliptic partial differential equations.

\subsection{An example to Theorem 1}

Let $\Omega \subset \mathbf{R}^{n}$ be a bounded domain with sufficiently smooth boundary $\partial \Omega$, and let $X=W_{0}^{1,2}(\Omega)$ be the Sobolov space with the norm $\|u\|=\left\{\int_{n}|\nabla u|^{2} d x\right\}^{1 / 2}$. We consider functionals on $W_{0}^{1,2}(\Omega)$ of the type

$$
\Phi(u)=\frac{1}{2} \int_{\Omega}|\nabla u|^{2} d x+\frac{1}{4} \int_{\Omega} u^{4} d x-\frac{1}{3} \int_{\Omega} u^{3} d x
$$

and use the following assumptions:

(P1) $p \in C(\bar{\Omega} \times \mathbf{R}, \mathbf{R})$.

(P2) There are constants $a, b \geq 0$ such that $|p(x, t)| \leq a+b|t|^{\bullet}$ for all $(x, t) \in \Omega \times \mathbf{R}$, where $0 \leq s<(n+2) /(n-2)$ if $n>2$ and $0 \leq s<+\infty$ if $n=1,2$.

We define $P(x, t)=\int_{0}^{t} p(x, z) d z$, where $(x, t) \in \Omega \times \mathbf{R}$, and consider the following functionals $\Phi$ and $\Upsilon$ on $W_{0}^{1,2}(\Omega)$ :

$$
\Upsilon(u)=\frac{1^{u}}{r+1} \int_{\Omega}|u|^{r+1} d x+\int_{\Omega} P(x, u) d x, \quad \Phi(u)=\frac{1}{2} \int_{\Omega}|\nabla u|^{2} d x+\Upsilon(u),
$$

where $s<r<(n+2) /(n-2)$ if $n>2$ and $s<r$ if $n=1,2$. To simplify our formulas we here and in the following write $\int_{\Omega} P(x, u) d x$ instead of $\int_{\Omega} P(x, u(x)) d x$. Furthermore, we set

$$
\begin{aligned}
& \mathcal{S}_{0}=\left\{Q \in C(\Omega \times \mathbf{R}, \mathbf{R}) \mid Q(x, t)=\int_{0}^{t} q(x, z) d z, q \in C_{b}(\widetilde{\Omega} \times \mathbf{R}, \mathbf{R})\right\}, \\
& \mathcal{S}=\left\{\Psi: W_{0}^{1,2}(\Omega) \rightarrow \mathbf{R} \mid \Psi(u)=\int_{\Omega} Q(x, u) d x, Q \in \mathcal{S}_{0}\right\} .
\end{aligned}
$$

It is our aim to prove that $\Phi$ and $\mathcal{S}$ satisfy all assumptions of Theorem 1 . For this, after the following remarks, we formulate two lemmas. 
Remarks: 1) If the assumptions of Theorem 1 are fulfilled, then $u \in$ crit $_{\boldsymbol{X}, c} \boldsymbol{\Phi}$ resp. $u \in \operatorname{crit} \dot{x}_{r_{*}} \Phi_{*}$ is a weak solution of the boundary value problem

resp.

$$
-\Delta u+u|u|^{r-1}+p(x, u)=0 \quad \text { in } \Omega, u=0 \text { on } \partial \Omega
$$

$$
-\Delta u+u|u|^{r-1}+p(x, u)+q(x, u)=0 \quad \text { in } \Omega, u=0 \text { on } 8 \Omega .
$$

2) For $\epsilon>0$ the condition $|\Psi(u)|<\epsilon$ for all $u \in X$, i. e. $\left|\int_{\Omega} Q(x, u) d x\right|<\epsilon$ for all $u \in X$, is fulfilled if, for example, $\sup _{(x, t) \in \Omega \times R} Q(x, t)<\epsilon|\Omega|^{-1}$.

Lemma 1 (cf. [5: Appendix B]): Under the assumptions stated above it holds (for arbitrary $\boldsymbol{\Psi} \in \mathcal{S})$ :

1) $\Phi, \Psi, \Upsilon \in C^{1}\left(W_{0}^{1,2}(\Omega), R\right)$, and for all $u, v \in W_{0}^{1,2}(\Omega)$ we have

$$
\begin{aligned}
& \left\langle\Phi^{\prime}(u), v\right\rangle=\int_{n} \nabla u \nabla v d x+\left\langle\Upsilon^{\prime}(u), v\right\rangle, \quad\left\langle\Psi^{\prime}(u), v\right\rangle=\int_{\Omega} q(x, u) v d x, \\
& \left\langle\Upsilon^{\prime}(u), v\right\rangle=\int_{\Omega} u|u|^{p-1} v d x+\int_{\Omega} p(x, u) v d x .
\end{aligned}
$$

2) $\Psi, \Upsilon: W_{0}^{1,2}(\Omega) \rightarrow \mathbf{R}$ are weakly continuous; $\Psi^{\prime}, \Upsilon^{\prime}: W_{0}^{1,2}(\Omega) \rightarrow\left[W_{0}^{1,2}(\Omega)\right]^{\prime}$ are compact.

Lemma 2: If (P1), (P2) hold, then $\Phi_{*}=\Phi+\Psi$ satisfies (PS) for each $\Psi \in \mathcal{S}$.

Remark: Since the functional $\Psi=0$ belongs to $\mathcal{S}$, Lemma 2 yields that $\Phi$ satisfies (PS).

Proof of Lemma 2: a) If $q \in C_{b}(\bar{\Omega} \times \mathbf{R}, \mathbf{R})$, then $p_{*}=p+q$ satisfies:

(P1). p. $\in C(\overline{\mathbf{\Omega}} \times \mathbf{R}, \mathbf{R})$.

(P2). There are constants $a_{*}, b_{*} \geq 0$ such that $\left|p_{*}(x, t)\right| \leq a_{*}+b_{*}|t|^{\bullet}$ for all $(x, t) \in \Omega \times \mathbf{R}$, where $s$ is the number from (P2).

The validity of (P1). is obvious, (P2). follows from $\left|p_{*}(x, t)\right| \leq|p(x, t)|+|q(x, t)| \leq a_{*}+b_{*}|t|^{0}$ with $a_{*}=a+\sup _{(x, t) \in \Pi \times R}|q(x, t)|, b_{*}=b$.

b) Let $\left(u_{m}\right) \subset W_{0}^{1,2}(\Omega)$ be a sequence such that $\left|\Phi_{*}\left(u_{m}\right)\right| \leq M<\infty$ for all $m \in N$ and $\Phi_{\bullet^{\prime}}\left(u_{m}\right) \rightarrow 0$ as $m \rightarrow \infty$. We show that this sequence is bounded in $W_{0}^{1,2}(\Omega)$. From (P2). there follows the existence of constants $A, B \geq 0$ such that $|P(x, t)+Q(x, t)| \leq A+B|t|^{0+1}$ for all $x \in \Omega$. The Hölder inequality yields for all $u \in W_{0}^{1,2}(\Omega)$

$$
\begin{aligned}
\Upsilon(u)+\Psi(u) & =\frac{1}{r+1} \int_{\Omega}|u|^{r+1} d x+\int_{\Omega}(P(x, u)+Q(x, u)) d x \\
& \geq \frac{1}{r+1} \int_{\Omega}|u|^{r+1} d x-A \int_{\Omega} d x-B \int_{\Omega}|u|^{\rho+1} d x \\
& \geq \frac{1}{r+1} \int_{\Omega}|u|^{r+1} d x-B|\Omega|^{\frac{r-1}{r+1}}\left\{\int_{\Omega}|u|^{r+1} d x\right\}^{\frac{c+1}{r+1}}-A|\Omega| \\
& =\|u\|_{r+1}^{++1}\left\{\frac{1}{r+1}\|u\|_{r+1}^{r-s}-B|\Omega|^{\frac{r-t}{r+1}}\right\}-A|\Omega| \geq C .
\end{aligned}
$$


Hence, for the sequence $\left(u_{m}\right)$ we have $M \geq \Phi_{\bullet}\left(u_{m}\right) \geq \frac{1}{2}\left\|u_{m}\right\|^{2}+C$, and, therefore, this sequence is bounded in $W_{0}^{1,2}(\Omega)$.

c) Let $D: W_{0}^{1,2}(\Omega) \rightarrow\left[W_{0}^{1,2}(\Omega)\right]^{\prime}$ be the duality mapping given by $\langle D u, v\rangle=\int_{\Omega} \nabla u \nabla v d x$ for all $u, v \in W_{0}^{1,2}(\Omega)$. Then, it holds

$$
D^{-1} \Phi_{*}^{\prime}(u)=u-D^{-1} \Upsilon^{\prime}(u)-D^{-1} \Psi^{\prime}(u)
$$

for each $u \in W_{0}^{1,2}(\Omega)$. The operators $D^{-1} \Upsilon^{\prime}$ and $D^{-1} \Psi^{\prime}$ are compact since $D^{-1}$ is continuous and $\Upsilon^{\prime}, \Psi^{\prime}$ are compact. The sequence $\left(u_{m}\right)$ is bounded, therefore, there exists a subsequence $\left(u_{m^{\prime}}\right) \subseteq\left(u_{m}\right)$ such that $\left(D^{-1} \Upsilon^{\prime}\left(u_{m^{\prime}}\right)\right)$ and $\left(D^{-1} \Psi^{\prime}\left(u_{m^{\prime}}\right)\right)$ are convergent in $W_{0}^{1,2}(\Omega)$. Furthermore, $D^{-1} \Phi_{*}^{\prime}\left(u_{m^{\prime}}\right) \rightarrow 0$ if $m^{\prime} \rightarrow \infty$, and (3) yields the convergence of $\left(u_{m^{\prime}}\right)$ d

Now, setting $\Psi=0$ in (2) we see that $\Phi(u) \geq \frac{1}{2}\|u\|^{2}+C$ for all $u \in W_{0}^{1,2}(\Omega)$. It follows that $\Phi$ is bounded below on $W_{0}^{1,2}(\Omega)$. So, for our example, all assumptions of Theorem 1 are satisfied.

\subsection{Critical values of mountain pass type}

In this subsection we assume:

(B1) $X$ is a real Banach space, $\Phi \in C^{1}(X, R)$.

(B2) There are positive constants $R$ and $\alpha$ such that $\Phi(u) \geq \alpha$ for all $u \in X$ with $\|u\|=R$.

(B3) There exists $u_{1} \in X$ with $\left\|u_{1}\right\|>R$ and $\Phi\left(u_{1}\right)<\alpha ; \Phi(0)<\alpha$.

(B4) $\mathcal{K}=\left\{p([0,1]) \mid p \in C([0,1], X), p(0)=0, p(1)=u_{1}\right\} ; c=\sup _{K \in \mathcal{K}} \sup _{u \in K} \Phi(u)$.

Using Ekeland's variational principle and the subdifferential calculus of convex functions, one gets the following result (cf. [3]).

Proposition 2s Assume (B1) to (B4) hold. Then given $\sigma>0$ there exists $u_{\circ} \in X$ such that $c \leq \Phi\left(u_{\sigma}\right) \leq c+\sigma$ and $\left\|\Phi^{\prime}\left(u_{\sigma}\right)\right\| \leq \sigma$.

Our perturbation result reads as follows.

Theorem 2: Suppose (B1) to (B4) hold. Let $S$ be a class of functionals $\Psi \in C^{1}(X, \mathbf{R})$ such that the functional $\Phi_{*}=\Phi+\Psi$ satisfies (PS) for all $\Psi \in \mathcal{S}$. Then for each $\epsilon>0$ there exists

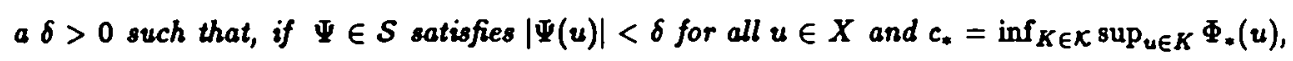
then $c_{*} \in(c-\epsilon, c+\epsilon)$ and crit $x_{x_{0}} \Phi_{*} \neq 0$.

Proof: Let $\epsilon>0$ be a given number, and set $\beta=\max \left\{\Phi(0), \Phi\left(u_{1}\right), 0\right\}$. It holds $\beta<\alpha$, by (B3). Set $\delta=\min \{\epsilon / 2,(\alpha-\beta) / 3\}$ and let $\Psi$ be a functional satisfying the assumptions of our theorem. Obviously $c_{*} \in[c-\delta, c+\delta] \subset(c-\epsilon, c+\epsilon)$, so that it remains to prove crit $x_{*} \Phi_{*} \Phi_{*} \neq 0$. 
Setting $\alpha_{*}=(\alpha+\beta) / 2$ we find $\Phi_{*}(0)=\Phi(0)+\Psi(0)<\beta+\delta \leq \beta+(\alpha-\beta) / 2<\alpha_{*}$, and analoguously $\Phi_{*}\left(u_{1}\right)<\alpha_{*}$. For $u \in X$ with $\|u\|=R$ it holds $\Phi_{*}(u)=\Phi(u)+\Psi(u)>a-\delta \geq$ $a-(\alpha-\beta) / 3>\alpha_{*}$. Now, we apply Proposition 2 to the functional $\Phi_{*}$. A repetition of the proof of Theorem 1 yields crit $x_{, c_{*}} \Phi_{*} \neq 0$ D

In the following, using the setting of the Example to Theorem 1, we give an application.

\subsection{An example to Theorem 2}

We consider functionals on $W_{0}^{1,2}(\Omega)$ of the type

$$
\Phi(u)=\frac{1}{2} \int_{n}|\nabla u|^{2} d x-\frac{1}{4} \int_{\Omega} u^{4} d x .
$$

To this end, we add to (P1), (P2) in the Example to Theorem 1 the following assumptions:

(P3) $p(x, t)=o(t)$ as $t \rightarrow 0$ for all $x \in \bar{\Omega}$.

(P4) There are constants $\mu>2, r \geq 0$ such that $0<\mu P(x, t) \leq t p(x, t)$ for all $|t| \geq r$ and $z \in \Omega$, where $P(x, t)=\int_{0}^{t} p(x, z) d z$.

Remarks: 1) If $n=1$, (P2) can be dropped while if $n=2$, it suffices that $|p(x, t)| \leq$ $\operatorname{axp}(\phi(t))$ for all $x \in \Omega$ where $\phi(t) t^{-2} \rightarrow 0$ as $|t| \rightarrow \infty$. 2) Integrating condition (P4) shows that there exist constants $a_{1}, b_{1}>0$ such that

$$
P(x, t) \geq a_{1}|t|^{\mu}-b_{1}
$$

for all $x \in \Omega$.

Let $S$ be as in the Example to Theorem 1, and consider the following functionals on $W_{0}^{1,2}(\Omega)$ :

$$
\Upsilon(u)=-\int_{\Omega} P(x, u) d x, \quad \Phi(u)=\frac{1}{2} \int_{\Omega}|\nabla u|^{2} d x+\Upsilon(u)
$$

Now, it is our aim to prove that $\Phi$ and $S$ satisfy all assumptions of Theorem 2. For this, after the following remarks, we premise a lemma.

Remarks: 1) Under the assumptions of Theorem 2, $u \in \operatorname{crit}_{X_{,},} \Phi$ resp. $u \in \operatorname{crit}_{X, c_{*}} \Phi_{*}$ is a weak solution of the boundary value problem

$$
-\Delta u-p(x, u)=0 \quad \text { in } \Omega, u=0 \text { on } 8 \Omega
$$

resp.

$$
-\Delta u-p(x, u)+q(x, u)=0 \quad \text { in } \Omega, u=0 \text { on } \partial \Omega .
$$

2) Cf. Remark 2) before Lemma 1. 3) Under our assumtions Lemma 1 stays true if we make the change $\left\langle Y^{\prime}(u), v\right\rangle=-\int_{\Omega} p(x, u) v d x$. 
Lemma 3: If (P1), (P2) and (P4) are satisfied, then $\Phi_{*}=\Phi+\Psi$ satisfies (PS) for all functionals $\Psi \in \mathcal{S}$.

Proof: a) If $q \in C_{b}(\bar{\Omega} \times \mathbf{R}, \mathbf{R})$, then $p_{*}=p-q$ satisfies (P1)., (P2), and the condition:

(P4). There are constants $\mu_{*}>2, r_{*} \geq 0$ such that $0<\mu_{*} P_{*}(x, t) \leq t p_{*}(x, t)$

for all $|t| \geq r_{*}$ and $x \in \Omega$ where $P_{*}(x, t)=\int_{0}^{t} p_{*}(x, z) d z$.

Indeed, the validity of (P1)., (P2). was proved in Lemma 2. To verify (P4), we choose an arbitrary number $\mu_{*} \in(2, \mu)$.

i) Using (4) we get

$$
\mu_{*} P_{*}(x, t)=\mu_{*} P(x, t)-\mu_{*} Q(x, t) \geq \mu_{*}\left(a_{1}|t|^{\mu}-|t| \sup _{(x, t) \in \Omega_{\times} \times \mathrm{R}} g(x, t)\right)-\mu_{*} b_{1} .
$$

Since $\mu>2$, there is an $r_{*}^{(1)}>0$ such that $\mu_{*} P_{*}(x, t)>0$ for $|t| \geq r_{*}^{(1)}$.

i) From (4) it follows that there exists $r_{*}^{(2)}>0$ such that for all $|t| \geq r_{*}^{(2)}$ it holds

Hence,

$$
\left(\mu-\mu_{*}\right) P(x, t) \geq\left(\mu-\mu_{*}\right)\left(a_{1}|t|^{\mu}-b_{1}\right) \geq\left(\mu_{*}+1\right)|t| \sup _{(x, t) \in \bigcap_{\times R}}|q(x, t)| .
$$

$$
\begin{aligned}
\mu_{*} P(x, t)-\mu_{*} Q(x, t)+t q(x, t) & \leq \mu_{*} P(x, t)+\mu_{*}|Q(x, t)|+|t||q(x, t)| \\
& \leq \mu_{*} P(x, t)+\left(\mu_{*}+1\right)|t| \sup _{(x, t) \in \Omega_{\times} \times \mathrm{R}}|q(x, t)| \\
& \leq \mu P(x, t)
\end{aligned}
$$

and using (P4) we obtain for $|t| \geq r_{*}^{(2)}$ :

$$
\mu_{*} P_{*}(x, t)=\mu_{*} P(x, t)-\mu_{*} Q(x, t) \leq \mu P(x, t)-t q(x, t) \leq t p_{*}(x, t) .
$$

If we set $r_{*}=\max \left\{r_{*}^{(1)}, r_{*}^{(2)}\right\}$, then from i), ii) it follows $0<\mu_{*} P_{*}(x, t) \leq t p_{*}(x, t)$ for all $|t| \geq r_{*}$ and $z \in \Omega$.

b) We show that each sequence $\left(u_{m}\right) \subset W_{0}^{1,2}(\Omega)$ such that $\left|\Phi_{*}\left(u_{m}\right)\right| \leq M<\infty$ for all $m \in N$ and $\Phi_{*}^{\prime}\left(u_{m}\right) \rightarrow 0$ as $m \rightarrow \infty$ is bounded. In fact, using (P1)., (P2), and (P4), we get for all $m \geq m_{0}, m_{0}$ sufficiently large:

$$
\begin{aligned}
M \geq & \Phi_{*}\left(u_{m}\right)=\frac{1}{2}\left\|u_{m}\right\|^{2}-\int_{\Omega} P_{*}\left(x, u_{m}\right) d x \\
= & \frac{1}{\mu_{*}}\left\{\left\|u_{m}\right\|^{2}-\int_{\Omega} p_{*}\left(x, u_{m}\right) u_{m} d x\right\}+\left(\frac{1}{2}-\frac{1}{\mu_{*}}\right)\left\|u_{m}\right\|^{2} \\
& -\int_{\Omega}\left[P_{*}\left(x, u_{m}\right)-\frac{1}{\mu_{*}} p_{*}\left(x, u_{m}\right) u_{m}\right] d x \\
= & \frac{1}{\mu_{*}}\left\langle\Phi_{*}^{\prime}\left(u_{m}\right), u_{m}\right\rangle+\left(\frac{1}{2}-\frac{1}{\mu_{*}}\right)\left\|u_{m}\right\|^{2}-\int_{\left\{u_{m} \geq r_{*}\right\}}\left[P_{*}\left(x, u_{m}\right)-\frac{1}{\mu_{*}} p_{*}\left(x, u_{m}\right) u_{m}\right] d x \\
& -\int_{\left\{u_{m}\left\langle r_{*}\right\}\right.}\left[P_{*}\left(x, u_{m}\right)-\frac{1}{\mu_{*}} p_{*}\left(x, u_{m}\right) u_{m}\right] d x \\
\geq & -\frac{1}{\mu_{*}}\left\|\Phi_{*}^{\prime}\left(u_{m}\right)\right\|\left\|u_{m}\right\|+\left(\frac{1}{2}-\frac{1}{\mu_{*}}\right)\left\|u_{m}\right\|^{2}+C \\
= & -\frac{1}{\mu_{*}}\left\|u_{m}\right\|+\left(\frac{1}{2}-\frac{1}{\mu_{*}}\right)\left\|u_{m}\right\|^{2}+C
\end{aligned}
$$


c) Now, it follows analogously to step c) of the proof of Lemma 2 that $\left(u_{m}\right)$ contains a convergent subsequence

To show that for our example all assumptions of Theorem 2 are satisfied it remains to verify (B1) to (B3).

(B1): This follows from Lemma 1.

(B2): From (P3) follows that for any $\epsilon>0$ there exists a $\delta>0$ such that

$$
|P(x, t)| \leq t^{2} / 2
$$

for all $x \in \bar{\Omega},|t|<\delta$. Furthermore, given any $\delta>0$, there exists an $A>0$ such that

$$
|P(x, t)| \leq A|t|^{0+1}
$$

for all $x \in \Omega,|t| \geq \delta$. To see this we observe that (P2) implies

$$
|P(x, t)| \leq\left|\int_{0}^{t}\left(a+b|z|^{0}\right) d z\right| \leq a|t|+\frac{b}{s+1}|t|^{\bullet+1}
$$

for all $(x, t) \in \Omega \times \mathbf{R}$, and (6) follows if we choose $A \geq a \delta^{-*}+b(s+1)^{-1}$. Inequalities (5) and (6) imply $|P(x, t)| \leq \epsilon t^{2} / 2+A|t|^{a+1}$ for all $z \in \Omega, t \in \mathbf{R}$. Hence, using the Sobolev imbedding $W_{0}^{1,2}(\Omega) \rightarrow L_{q}(\Omega)$ for $q(n-2)<2 n$ we have for $u \in W_{0}^{1,2}(\Omega)$

$$
|\Upsilon(u)| \leq \frac{\epsilon}{2} \int_{\Omega} u^{2} d x+A \int_{\Omega}|u|^{e+1} d x \leq C_{1}\left(\frac{\epsilon}{2}+A\|u\|^{e-1}\right)\|u\|^{2}
$$

with a positive constant $C_{1}$, depending only on $\Omega$. It follows

$$
|\Phi(u)| \geq \frac{1}{2}\|u\|^{2}-C_{1}\left(\frac{\epsilon}{2}+A\|u\|^{s-1}\right)\|u\|^{2}=\left(\frac{1}{2}-C_{1} \frac{\epsilon}{2}\right)\|u\|^{2}-C_{1} A\|u\|^{\rho+1} .
$$

If we choose $\epsilon \in\left(0, C_{1}^{-1}\right)$, then there are positive constants $R$ and $\alpha$ such that $\left(1 / 2-C_{1} \epsilon / 2\right) R^{2}-$ $C_{1} A R^{\rho+1} \geq \alpha$. For $u \in W_{0}^{1,2}(\Omega)$ with $\|u\|=R$ relation $(7)$ yields $\Phi(u) \geq a$.

(B3): Obviously it holds $\Phi(0)=0<a$. Let $u \in W_{0}^{1,2}(\Omega)$ be arbitrary such that $u \neq 0$. Then for all $t \in \mathbf{R}$ we have

$$
\begin{aligned}
\Phi(t u) & =\frac{t^{2}}{2}\|u\|^{2}-\int_{\Omega} P(x, t u) d x \leq \frac{t^{2}}{2}\|u\|^{2}-a_{1} \int_{\Omega}|t u|^{\mu} d x+b_{1}|\Omega| \\
& \leq \frac{t^{2}}{2}\|u\|^{2}-a_{1}|t|^{\mu} \int_{\Omega}|u|^{\mu} d x-b_{1}|\Omega|
\end{aligned}
$$

where we did use (4). Because of $\mu>2$ it follows $\Phi(t u) \rightarrow-\infty$ as $|t| \rightarrow \infty$, and (B3) is satisfied.

\section{Perturbation of local extrema with smooth side conditions}

In this section we consider the perturbation of critical values of functionals which are restricted to manifolds. First of all we state an abstract perturbation principle. 
2.1. General perturbation principle for critical values of maximum-minimum type Let us consider a real Banach space $X$ and a functional $\Phi: M \subseteq X \rightarrow \mathbf{R}$. For a fixed real number $c$ we denote by $\operatorname{crit}_{M, c} \Phi$ a certain subset of $M$ (in our applications this will be the set of critical points of the restriction of the functional $\Phi$ to the manifold $M$ ).

We now formulate the following hypotheses.

(H1) $\Phi: M \subseteq X \rightarrow \mathbf{R}$ is a functional on the real Banach space $X ; M \neq 0$.

(H2) $\mathcal{K}$ is a non-empty class of non-empty subsets of $M$. For $c$ defined by

$$
c=\sup _{K \in \mathcal{K}} \inf _{u \in K} \Phi(u)
$$

it holds $c \neq \pm \infty$.

(H3) $\mathcal{D}$ is a subset of $C(M, M)$ such that $\mathcal{X}$ is invariant under $\mathcal{D}$, i. e. $d \in \mathcal{D}$ and $K \in \mathcal{X}$ implie $d(K) \in \mathcal{K}$.

(B4) If crit $_{M, c} \Phi=0$, then there exists a real number $\epsilon>0$ and a mapping $d \in \mathcal{D}$ such that

$$
\Phi(u) \geq c-\epsilon, u \in M, \text { implies } \Phi(d(u)) \geq c+\epsilon .
$$

Proposition 3: With the assumtions (H1) to (H4), crit $_{M, c} \Phi \neq 0$.

Proof (Cf. [10: Chapter 44.2]): Let us assume that $\mathrm{crit}_{M, c} \Phi=0$. By (H4), there exist $\epsilon>0$ and $d \in \mathcal{D}$ such that (9) holds. We choose $K \in \mathcal{X}$ for which $\inf _{u \in K} \Phi(u) \geq c-\epsilon$, thus $\inf _{u \in d(K)} \Phi(u) \geq c+\epsilon$. Due to (H3), $d(K) \in \mathcal{K}$, i. e. , inf $u \in d(K) \Phi(u) \leq c$ by (8). This is a contradiction

Remarks In the applications it is possible to choose for $\mathcal{D}$ in (H3) the set of all homeomorphisms of $M$ onto itself.

We now assume (H1) to (H4) and put the question if for a perturbed functional $\Phi_{*}=\Phi+\Psi$

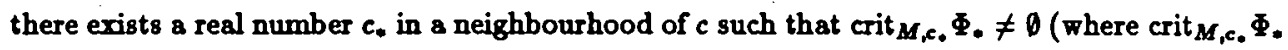
is defined analogously to crit ${ }_{M, e} \Phi$ by formally replacing $c, \Phi$ by $c_{*}, \Phi_{*}$, respectively). To this end, we formulate in addition to (H1) - (H4) the following hypotheses:

(H5) $\Psi: M \subseteq X \rightarrow \mathbf{R}$ is a functional and $\delta>0$ is a real number such that $|\Psi(u)|<\delta$ for all $u \in M$.

(H6) $\mathcal{K}_{*} \subseteq \mathcal{K}$ is non-empty. For the functional $\Phi_{*}=\Phi+\Psi$ we define

$$
c_{*}=\sup _{K \in K_{*}} \inf _{u \in K} \Phi_{*}(u) \text {. }
$$

(B7) There exists a real number $\sigma>0$ and a set $K_{\sigma} \in \mathcal{K}_{*}$ such that

$$
\inf _{u \in K_{e}} \Phi(u)>c-\sigma .
$$


(H8) $\mathcal{D}_{*}$ is a subset of $C(M, M)$ ouch that $\mathcal{K}_{*}$ is invariant under $\mathcal{D}_{*}$.

(H8) If crit $M_{M_{1} c_{*}} \Phi_{*}=0$, then there exists a real number $\epsilon_{*}>0$ and a mapping $d_{*} \in \mathcal{D}_{*}$ such that

$$
\Phi_{*}(u) \geq c_{*}-\epsilon_{*}, u \in M, \text { implies } \Phi_{*}\left(d_{*}(u)\right) \geq c_{*}+\epsilon_{*}
$$

Proposition 4: With the assumptions (H1) to (H7), it holds

$$
c \in(c-\delta-\sigma, c+\delta] \text {. }
$$

Proof: By (H5), (H6), we have

$$
c . \leq \sup _{K \in \mathcal{K}} \inf _{u \in K} \Phi_{*}(u) \leq \sup _{K \in \mathcal{K}} \inf _{u \in K} \Phi(u)+\delta \leq c+\delta .
$$

On the other hand, from (H7) it follows that

$$
c_{*} \geq \inf _{u \in K_{\sigma}} \Phi_{*}(u) \geq \inf _{u \in K_{*}} \Phi(u)-\delta>c-\sigma-\delta \mathbf{D}
$$

Proposition 5: If (H1) to (H6) hold and if $\mathcal{K}_{*}=\mathcal{K}$, then $c_{*} \in[c-\delta, c+\delta]$.

Proof: Since $\mathcal{K}_{*}=\mathcal{K}$, for every real number $\sigma>0$ there is a set $K_{\sigma} \in \mathcal{X}$ such that (11) holds. Now, the assertion follows from Proposition 4

Proposition 6: With the assumptions (H1) to (H9), $\mathrm{crit}_{M, c} \Phi_{*} \neq \emptyset$.

The proof is a repetition of that of Proposition $3 \mathbf{0}$

Corollary 2: Propositions 1 to $f$ remain true if (8) to (13) are replaced, respectively, by

$$
\begin{gathered}
c=\inf _{K \in \mathcal{K}} \sup _{u \in K} \Phi(u), \\
\Phi(u) \leq c+\epsilon, u \in M, \text { implies } \Phi(d(u)) \leq c-\epsilon, \\
c_{*}=\inf _{K \in K_{*}} \sup _{u \in K} \Phi_{*}(u), \\
\sup _{u \in K_{\sigma}} \Phi(u)<c+\sigma, \\
\Phi_{*}(u) \leq c_{*}+\epsilon_{*}, u \in M, \text { implies } \Phi_{*}\left(d_{*}(u)\right) \leq c_{*}-\epsilon_{*}, \\
c_{*} \in[c-\delta, c+\delta+\sigma) .
\end{gathered}
$$

Remark: Im many applications of the perturbation principle it is possible to choose $\mathcal{K}_{*}=\mathcal{K}$ in (H6). This occurs in Subsection 2.3. But it could be necessary to choose a class $\mathcal{X}$ (e. g. , to get more information on the number of critical values) such that it is not possible to take $\mathcal{K}_{*}=\mathcal{K}$, because the mappings $d_{\text {. from }}(\mathrm{H} 9)$ do not guarantee $d_{*}(K) \in \mathcal{K}$ for all $K \in \mathcal{K}$. This is the case in Subsection 2.5. 


\subsection{Stability of the Local Palais-Smale Condition}

In this subsection we suppose the following assumptions:

(C1) $X$ is a real reflexive Banach space, $\Phi, \Upsilon \in C^{1}(X, R)$.

(C2) $\alpha$ is a fixed real number, $M=\{u \in X \mid \Upsilon(u)=\alpha\}$.

(C3) There exists a continuous function $\nu: M \rightarrow X$ such that $\|\nu(u)\|=1$ for all $u \in M$ and $\inf _{u \in K}\left|\left\langle\Upsilon^{\prime}(u), \nu(u)\right\rangle\right|>0$ for each bounded set $K \subset M$.

(C4) $\Upsilon^{\prime}: X \rightarrow X^{\prime}$ is bounded and locally Lipschitz continuous on $M$.

(C5) There is a set $\dot{M} \supset M$ which is closed with respect to the weak convergence in $X$ such that $\Phi^{-1}(B) \cap \dot{M}$ is bounded for each bounded set $B \subset \mathbf{R}$.

We define for $u \in X$

$$
\bar{\Phi}^{\prime}(u)=\Phi^{\prime}(u)-\lambda(u) \Upsilon^{\prime}(u), \quad \lambda(u)=\frac{\left\langle\Phi^{\prime}(u), \nu(u)\right\rangle}{\left\langle\Upsilon^{\prime}(u), \nu(u)\right\rangle}
$$

We want to study the sets

$$
\operatorname{crit}_{M, c} \Phi=\left\{u \in M \mid \tilde{\Phi}^{\prime}(u)=0, \Phi(u)=c\right\}, c \in \mathbf{R}
$$

Remark: If $u \in \operatorname{crit}_{M, c} \Phi$, then it holds $\Phi^{\prime}(u)=\lambda \Upsilon^{\prime}(u)$ with a real number $\lambda, i$. e. , $u$ is a critical point of the restriction of the functional $\Phi$ to the manifold $M$.

Let $c$ be a fixed number. The functional $\Phi$ is said to satisfy a local Palais-Smale condition (PS) $)_{e}$ on $M$ if the following holds:

(PS) Every sequence $\left(u_{m}\right) \subset M$ with $\Phi\left(u_{m}\right) \rightarrow c$ and $\bar{\Phi}^{\prime}\left(u_{m}\right) \rightarrow 0$ as $m \rightarrow \infty$ has a strangly convergent subsequence.

We need the following so-called Deformation Theorem which here is formulated as

Lemma 4: Assume that (C1) to (C5) are sativfied and that $\Phi$ satisfies $(\mathrm{PC})_{c}$ on $M, c \in \mathbf{R}$. If crit $M_{r} \Phi=0$, there exist $\epsilon>0$ and $d \in C(M \times[0,1], M)$ such that the following hold:

1) The mapping $u \mapsto d(u, t)$ is a homeomorphism of $M$ onto itself for all $t \in[0,1]$.

2) $d(u, 0)=u$ for all $u \in M$.

3) $\Phi(d(u, t)) \geq \Phi(u)$ for all $u \in M, t \in[0,1]$.

4) $\Phi(u) \geq c-\epsilon, u \in M$ implies $\Phi(d(u, 1)) \geq c+\epsilon$.

In [9: Proposition 1], the Proof is given for the case that $\nu(u)=u /\|u\|$ satisfies (C3), and that of Lemma 4 is completely analogous a 
Lemma 5: (PS) $)_{c}$ holds under the following hypotheses.

a) Assumptions (C1) to (C5) are satisfied.

b) $\Upsilon^{\prime}$ satisfies (S) 1 .

c) $\Phi^{\prime}: X \rightarrow X^{\prime}$ is strongly continuous.

d) If $u_{m} \rightarrow u$ as $m \rightarrow \infty$, $\left(u_{m}\right) \subset M$, and $\Phi(u)=c$, then $\Phi^{\prime}(u) \neq 0$.

Proof: Let $\left(u_{m}\right) \subset M$ such that $\Phi\left(u_{m}\right) \rightarrow c$ and $\bar{\Phi}^{\prime}\left(u_{m}\right) \rightarrow 0$ as $m \rightarrow \infty$. This sequence is bounded, by (C5); therefore, there exists a subsequence $\left(u_{m^{\prime}}\right) \subseteq\left(u_{m}\right)$ such that $u_{m n^{\prime}} \rightarrow u_{0}$ as $m \rightarrow \infty$. (C1) and c) implie that the functional $\Phi$ is weakly continuous, and it follows $\Phi\left(u_{m^{\prime}}\right) \rightarrow \Phi\left(u_{0}\right)$ as $m^{\prime} \rightarrow \infty$ and $\Phi\left(u_{0}\right)=c$. Now, $\Phi^{\prime}\left(u_{0}\right) \neq 0$, by d).

The sequence $\left(\lambda\left(u_{m^{\prime}}\right)\right)$ is bounded because of (C3) and $\left.c\right)$, hence there is a further subsequence $\left(u_{m^{\prime \prime}}\right) \subseteq\left(u_{m^{\prime}}\right)$ such that $\lambda\left(u_{m^{\prime \prime}}\right) \rightarrow \lambda_{0}$ as $m^{\prime \prime} \rightarrow \infty$. It holds $\lambda_{0} \neq 0$. Otherwise, because of $\bar{\Phi}^{\prime}\left(u_{m^{\prime \prime}}\right)=\Phi^{\prime}\left(u_{m^{\prime \prime}}\right)-\lambda\left(u_{m^{\prime \prime}}\right) \Upsilon^{\prime}\left(u_{m^{\prime \prime}}\right), \bar{\Phi}^{\prime}\left(u_{m^{\prime \prime}}\right) \rightarrow 0$ as $m^{\prime \prime} \rightarrow \infty$ and the boundedness of $\left(\Upsilon^{\prime}\left(u_{m^{\prime \prime}}\right)\right)$ (by (C4)), $\lambda_{0}=0$ would imply $\Phi^{\prime}\left(u_{m^{\prime \prime}}\right) \rightarrow 0$ as $m^{\prime \prime} \rightarrow \infty$, which is a contradiction to $\Phi^{\prime}\left(u_{0}\right) \neq 0$.

For $m^{\prime \prime}$ sufficiently large it holds $\Upsilon^{\prime}\left(u_{m^{\prime \prime}}\right)=\lambda\left(u_{m^{\prime \prime}}\right)^{-1}\left(\Phi^{\prime}\left(u_{m^{\prime \prime}}\right)-\tilde{\Phi}^{\prime}\left(u_{m^{\prime \prime}}\right)\right)$, and from $\left.c\right)$ and $\tilde{\Phi}^{\prime}\left(u_{m^{\prime \prime}}\right) \rightarrow 0$ as $m^{\prime \prime} \rightarrow \infty$ it follows that the sequence $\left(\Upsilon^{\prime}\left(u_{m^{\prime \prime}}\right)\right)$ is convergent. Hence $u_{m \prime \prime} \rightarrow u_{0}$ as $m^{\prime \prime} \rightarrow \infty$, by b) 0

Now, we consider perturbations $\Phi_{\bullet}=\Phi+\Psi$ of the functional $\Phi$. The key is to guarantee that $\Phi_{*}$ satisfies (PS) $c_{\text {. }}$ for all $c_{*}$ in a neighbourhood of $c$. For that, assumption d) in Lemma 5 is to weak. This motivates the following considerations.

Lemma 6: Assume:

a) Assumptions (C1) to (C5) are satisfied.

b) $\mathbf{Y}^{\prime}$ satisfies $(\mathbf{S})_{1}$.

c) $\Phi^{\prime}$ is strongly continuous on $X$.

d) $c_{2}, c_{2} \in \mathbf{R} \cup\{ \pm \infty\}$ are such that $\tau=\inf \left\{\left\|\Phi^{\prime}(u)\right\| \mid u \in \tilde{M} \cap \Phi^{-1}\left(\left(c_{1}, c_{2}\right)\right)\right\}>0$.

e) $\delta$ is a positive real number such that $\delta<\tau$.

f) $\Psi \in C^{1}(X, \mathbf{R})$ has a strongly continuous derivative $\Psi^{\prime}$, and it holds for all $u \in \bar{M}$ :

$$
|\mathbf{\Psi}(u)|+\left\|\Psi^{\prime}(u)\right\|<\delta
$$

Then, it holds:

1) The functional $\Phi$ satisfies (PS) for every $c \in\left(c_{1}, c_{2}\right)$.

2) The functional $\Phi_{\bullet}=\Phi+\Psi$ satisfies (PS) for every $c \in\left(c_{1}+\delta, c_{2}-\delta\right)$. 
Proof: 1) follows immediately from Lemma 5.

2) We show that the perturbed functional $\Phi_{*}$ satisfies the assumptions of Lemma 5 for every number $c \in\left(c_{1}+\delta, c_{2}-\delta\right)$.

a) (C1) to (C4) are satisfied, it remains to verify (C5). Let $B \subset \mathbf{R}$ be a bounded set, then there exist real numbers $U, V$ such that $B$ lies in the interval $(U, V)$. Inequality (14) implies

$$
\Phi_{*}^{-1}(B) \cap \tilde{M} \subset \Phi_{*}^{-1}((U, V)) \cap \tilde{M} \subset \Phi^{-1}((U-\delta, V+\delta)) \cap \tilde{M}
$$

and the last set is bounded, by (C5).

b) Assumptions b) and c) of Lemma 5 are obviously satisfied.

c) To verify d) from Lemma 5 , let $\left(u_{m}\right) \subset M$ be such that $u_{m} \rightarrow u$ as $m \rightarrow \infty$ and $\Phi_{*}(u)=c, c \in\left(c_{1}+\delta, c_{2}-\delta\right)$. It follows $u \in \bar{M}$, and (14) implies $\Phi(u) \in\left(c_{1}, c_{2}\right)$. From the definition of $\tau$ in d) it follows $\left\|\Phi^{\prime}(u)\right\| \geq \tau$. Hence $\left\|\Phi_{*}^{\prime}(u)\right\| \geq\left\|\Phi^{\prime}(u)\right\|-\delta \geq \tau-\delta>0$, i. e. , $\Phi^{\prime}(u) \neq 0$ v

\subsection{Functionals on bounded level sets I}

We make the following assumptions:

(D1) $X$ is a real reflexive Banach space, $\operatorname{dim} X=\infty ; \Phi, \Upsilon \in C^{1}(X, \mathbf{R})$.

(D2) $\alpha \neq 0$ is a fixed real number, $M=\{u \in X: \Upsilon(u)=\alpha\}$ is non-empty. The set $\{u \in X: Y(u) \leq a\}$ is bounded; there is $u_{1} \in X, u_{1} \neq 0$, such that $\Upsilon\left(u_{1}\right)<\alpha, \Upsilon\left(-u_{1}\right)<\alpha$. It holds $\Upsilon(0)=0$.

(D3) There exists a continuous function $\nu: M \rightarrow X$ such that $\|\nu(u)\|=1$ for all $u \in M$ and $\inf _{u \in M}\left|\left\langle\Upsilon^{\prime}(u), \nu(u)\right\rangle\right|>0$.

(D4) $Y^{\prime}: X \rightarrow X^{\prime}$ satisfies (S), and is bounded and locally Lipschitz continuous on $M$.

(D5) There is a bounded set $\bar{M} \supset M$ which is closed with respect to the weak convergence in $X$.

(D6) $\Phi^{\prime}: X \rightarrow X^{\prime}$ is strongly continuous. $\Phi(u) \geq 0$ for all $u \in M$ and $\Phi$ is bounded on $M$. For $u \in \bar{M}$ it holds $\Phi(u)=0 \Longleftrightarrow u=0 \Longleftrightarrow \Phi^{\prime}(u)=0$.

Theorem 3: With the assumptions (D1) to (D6) the following assertions hold:

1) There exists a sequence $\left(c_{k}\right)$ of real numbers $c_{k}>0$ such that crit $M_{, c_{k}} \Phi \neq \emptyset$ for $k \geq 1$.

2) For every $k \geq 1$ and $\epsilon>0$ there exists $\delta>0$ such that for all $\Psi \in C^{1}(X, \mathbf{R})$ with strongly continuous derivative $\Psi^{\prime}$ and $|\Psi(u)|+\left\|\Psi^{\prime}(u)\right\|<\delta$ for all $u \in \tilde{M}$ there is a number $c_{*} \epsilon\left(c_{k}-\epsilon, c_{k}+\epsilon\right)$ for which crit $M_{M_{*} c_{*}} \Phi_{*} \neq 0$ (where $\left.\Phi_{*}=\Phi+\Psi\right)$. 
Remark: In 1) it may happen that all numbers $c_{k}$ are equal to each other.

Before we are able to prove Theorem 3 we need some preparations. For every $k \in \mathbb{N}$ we define

$$
\mathcal{K}=\left\{\begin{array}{l|l}
K \subset M & \begin{array}{l}
\text { There is an } e \in \mathbf{R}^{k}, e \neq 0 \text { such that } K \text { is homeomorphic } \\
\text { to the boundary of an open bounded neighbourhood of the } \\
\text { set }\{e,-e\} \text { in } \mathbf{R}^{k} \backslash\{0\}
\end{array}
\end{array}\right\}
$$

Lemma 7: Assume (D1) to (D6). Then $\mathcal{K}_{k} \neq \emptyset$ for all $k \in \mathrm{N}$.

Proof: Let $\left(u_{m}\right), m \geq 1$, be a sequence of linearly independent elements of $X$, where $u_{1}$ is taken as in (D2). For $k \in \mathbf{N}$, the subspace $E_{k}=\operatorname{span}\left\{u_{1}, \ldots, u_{k}\right\}$ is isomorphic to $\mathbf{R}^{k}$ by the canonical isomorphism $\psi_{k}: E_{k} \rightarrow \mathbf{R}^{k}$. The set $V=\left\{u \in E_{k}: \Upsilon(u)<\alpha\right\}$ is bounded by (D2) and contains $u_{1}$ and $-u_{1}$. Its boundary satisfies $\partial V \subset M$ since $\Upsilon$ is continuous. Furthermore, $\partial V=\psi_{k}^{-1}\left(\partial\left(\psi_{k}(V)\right)\right.$, and $\psi_{k}(V)$ is an open bounded neighbourhood of $\left\{\psi_{k}\left(u_{1}\right),-\psi_{k}\left(u_{1}\right)\right\}$ in $\mathbf{R}^{k} \backslash\{0\}$. Hence, $\partial V \in \mathcal{K}_{k}$.

Now we define for $K \in \mathbf{N}$

$$
c_{k}=\sup _{K \in K_{k}} \inf _{u \in K} \Phi(u)
$$

Lemma 8: With the assumptions (D1) to (D6), $0<c_{k}<+\infty$ for $k \in \mathbf{N}$.

Proof: Let $k \in \mathrm{N}$. By Lemma 7, there is a $K_{0} \in \mathcal{K}_{k}$. The set $K_{0}$ is compact, and $\Phi(u)>0$ for $u \in M$ (by (D2), (D6)), hence inf $u \in K_{0} \Phi(u)>0$. It follows $c_{k}>0$. Furthermore, $c_{k}<+\infty$, since $\Phi$ is bounded on $M$, by (D6) $D$

Proof of Theorem 3/1): We show that for $\mathcal{K}_{k}, c_{k}, k \in \mathrm{N}$, assumptions (H1) to (H4) of Subsection 2.1 are satisfied. Then, the assertion follows from Proposition 3.

(H1) is trivially satisfied, and (H2) follows from Lemma 7 and 8.

(H3): If we choose $\mathcal{D}=\operatorname{Hom}(M, M)$ (the set of all homeomorphisms of $M$ onto itself), then it is clear that $\mathcal{K}_{k}$ is invariant under $\mathcal{D}$.

(H4): Under the assumptions (D1) to (D6), all assumptions of Lemms 5 for $c=c_{k}$ are satisfied. Especially, since $c_{k}>0$ by Lemms 8, (D6) implies assumption d) of Lemms 5. Hence, $\Phi$ satisfies (PS) $c_{c_{k}}$, and Lemma 4 implies that the mapping $d(\cdot, 1)$ fulfills (H4) 1

We set for fixed $k \in \mathbf{N}$

$$
\tau=\inf \left\{\left\|\Phi^{\prime}(u)\right\| \mid u \in \dot{M}, \Phi(u) \in\left(c_{k} / 2,3 c_{k} / 2\right)\right\}
$$


Lemma 9: Suppose that (D1) to (D6) hold. Then $\tau>0$.

Proof: We give a proof by contradiction, by supposing $\tau=0$. Then, there is a sequence $\left(u_{m}\right) \subset \dot{M}$ such that $\Phi\left(u_{m}\right) \in\left(c_{k} / 2,3 c_{k} / 2\right)$ for $m \in N$ and $\left\|\Phi^{\prime}\left(u_{m}\right)\right\| \rightarrow 0$ as $m \rightarrow \infty$. Since $M$ is bounded there exists a subsequence $\left(u_{m^{\prime}}\right) \subseteq\left(u_{m}\right)$ such that $u_{m^{\prime}}-u \in \bar{M}$ as $m^{\prime} \rightarrow \infty$. The operator $\Phi^{\prime}$ is strongly continuous (and hence $\Phi$ is weakly continuous), therefore, $\Phi(u) \in\left[c_{k} / 2,3 c_{k} / 2\right]$ and $\Phi^{\prime}(u)=0$, in contradiction to (D6) 0

Proof of Theorem 3/2): Let $k \in N$ be fixed, and let $\epsilon>0$ be given. We assume $\epsilon<c_{h} / 2$. Let $\tau>0$ be the number defined in (15). Now, we choose $\delta>0$ such that

$$
\delta<\min \left\{\epsilon, \tau, c_{k} / 4\right\}
$$

Let $\Psi \in C^{1}(X, \mathbf{R})$ such that $\Psi^{\prime}$ is strongly continuous and $|\Psi(u)|+\left\|\Psi^{\prime}(u)\right\|<\delta$ for all $u \in \dot{M}$. It is our aim to apply Propositions 5 and 6, so we have to verify (H5) to (H9). To this end, we set $\mathcal{K}_{*}=\mathcal{K}_{k}$ and $\mathcal{D}_{*}=\mathcal{D}$.

(H5) to (H8) are obviously satisfied.

(H9): From Proposition 5 it follows $c_{*} \in\left[c_{k}-\delta, c_{k}+\delta\right]$. If we set in Lemma $6 c_{1}=c_{k} / 2$, $c_{2}=3 c_{k} / 2$, then we see that the functional $\Phi$ satisfies (PS) for every $c \in\left(c_{k} / 2+\delta, 3 c_{k} / 2-\delta\right)$, by Lemma 9. It holds $\left[c_{k}-\delta, c_{k}+\delta\right] \subset\left(c_{k} / 2+\delta, 3 c_{k} / 2-\delta\right)$, by (16), and therefore the functional $\Phi$. satisfies (PS) $c_{*}$. Now, from Lemma 4 it follows that (H9) is fulfilled.

From Proposition 6 it follows crit $M_{, c_{*}} \Phi_{*} \neq \emptyset$, and $c_{*} \in\left(c_{k}-\epsilon, c_{k}+\epsilon\right)$ because we have $c_{*} \in\left[c_{k}-\delta, c_{k}+\delta\right]$ and $(16)$ d

As an application of Theorem 3 we consider the following example.

\subsection{An example to Theorem 3}

Let $\Omega \subset \mathbf{R}^{n}, n \leq 3$ and $X=W_{0}^{1,2}(\Omega)$ as in the Example to Theorem 1. We consider the following functionals on $W_{0}^{1,2}(\Omega)$ :

$$
\begin{array}{ll}
\Upsilon(u)=\frac{1}{2} \int_{\Omega}|\nabla u|^{2} d x+\Gamma(u), & \Gamma(u)=-\frac{\lambda}{2} \int_{\Omega} u^{2} d x+\frac{1}{4} \int_{\Omega} u^{4} d x, \\
\Phi(u)=\frac{1}{q} \int_{\Omega}|u|^{q} d x, & \Psi(u)=\sigma \int_{\Omega} P(x, u) d x,
\end{array}
$$

where $0<q<6, \sigma \in \mathbf{R}, P(x, t)=\int_{0}^{t} p(x, z) d z$ for $(x, t) \in \Omega \times \mathbf{R}$ and $p$ satisfies (P1), (P2) (cf. Example to Theorem 1). The number $\lambda$ in the functional $\Gamma$ will be specified below. To show that Theorem 3 applies to this example, after the following remark, we premise three lemmas.

Remark: If we assume that the assumptions of Theorem 3 are fulfilled, then $u \in$ crit $_{M, c} \Phi$ 
resp. $u \in \operatorname{crit}_{M, c .} \Phi$. is a weak solution of the eigenvalue problem

resp.

$$
\begin{array}{ll}
\mu\left(-\Delta u-\lambda u+u^{3}\right)=u|u|^{q-2} & \text { in } \Omega, u=0 \text { on } \partial \Omega \\
\mu\left(-\Delta u-\lambda u+u^{3}\right)=u|u|^{q-2}+\sigma p(x, u) & \text { in } \Omega, u=0 \text { on } \delta \Omega .
\end{array}
$$

We consider the linear eigenvalue problem

$$
-\Delta \boldsymbol{u}=\lambda \boldsymbol{u} .
$$

It is well known that (17) possesses a sequence of eigenvalues $\left(\lambda_{m}\right)$ with $0<\lambda_{1}<\lambda_{2} \leq \ldots$ and $\lambda_{m} \rightarrow+\infty$ as $m \rightarrow \infty$. For the parameter $\lambda$ in $\Gamma$ we assume

$$
\lambda_{1}<\lambda<\lambda_{2} .
$$

Before we choose the number $\alpha$ we prove

Lemma 10: The functional $\Upsilon$ is bounded below on $W_{0}^{1,2}(\Omega)$ and $\inf _{u \in W_{0}^{1,2}(n)} \Upsilon(u)<0$.

Proof: a) Using the Hölder inequaltity and the Sobolev imbedding $W_{0}^{1,2}(\Omega) \rightarrow L_{4}(\Omega)$ we get the inequality

$$
\Upsilon(u) \geq \frac{1}{2}\|u\|^{2}-\frac{\lambda}{2}|\Omega|^{1 / 2}\|u\|_{4}^{2}+\frac{1}{4}\|u\|_{4}^{4} \geq \frac{1}{2}\|u\|^{2}+C
$$

for $u \in W_{0}^{1,2}(\Omega)$, i. e. $\Upsilon$ is bounded below on $W_{0}^{1,2}(\Omega)$.

b) Let $\tilde{u}$ be an eigensolution of (17) to the first eigenvalue $\lambda_{1}$, hence $\bar{u} \in W_{0}^{1,2}(\Omega)$, $\bar{u} \neq 0, \int_{\Omega}|\nabla \bar{u}|^{2} d x=\lambda_{1} \int_{\Omega} \tilde{u}^{2} d x$. We study the functional $\Upsilon$ along the one-dimensional subspace $\operatorname{span}\{\bar{u}\}$. For $t \in \mathbf{R}$ we set

where

$$
v(t)=\Upsilon(t \bar{u})=A t^{4}+B t^{2},
$$

$$
A=\frac{1}{4} \int_{\Omega} \tilde{u}^{4} d x, \quad B=\frac{1-\lambda / \lambda_{1}}{2} \int_{\Omega}|\nabla \tilde{u}|^{2} d x .
$$

From (18) it follows $B<0$, consequently there is a $t_{0} \in \mathbf{R}$ such that $v\left(t_{0}\right)<0$. Hence $\inf _{u \in W_{0}^{2}(\Omega)} \Upsilon(u)<0$ I

Now, we choose a number $\alpha$ such that

$$
\inf _{u \in W_{0}^{1,2}(\Omega)} \Upsilon(u)<\alpha<0
$$

Analogously to Lemma 1 we have

Lemma 11 (cf. [5] and [10: Corollary 26.14]): Under the assumptions stated above it holds:

1) $\Upsilon, \Phi, \Gamma, \Psi \in C^{1}\left(W_{0}^{1,2}(\Omega), \mathbf{R}\right)$ and for all $u, v \in W_{0}^{1,2}(\Omega)$ we have

$$
\begin{array}{ll}
\left\langle\Upsilon^{\prime}(u), v\right\rangle=\int_{\Omega} \nabla u \nabla v d x+\left\langle\Gamma^{\prime}(u), v\right\rangle, & \left\langle\Gamma^{\prime}(u), v\right\rangle=-\lambda \int_{\Omega} u v d x+\int_{n} u^{3} v d x, \\
\left\langle\Phi^{\prime}(u), v\right\rangle=\int_{\Omega} u|u|^{p-2} v d x, & \left\langle\Psi^{\prime}(u), v\right\rangle=\int_{\Omega} q(x, u) v d x .
\end{array}
$$


2) $\Phi, \Gamma, \Psi: W_{0}^{1,2}(\Omega) \rightarrow \mathbf{R}$ are weably continuous, $\Phi^{\prime}, \Gamma^{\prime}, \Psi^{\prime}: W_{0}^{1,2}(\Omega) \rightarrow\left[W_{0}^{1,2}(\Omega)\right]^{\prime}$ are strongly continuous (and hence compact).

It will be important that we know the exact number of critical points of $\Upsilon$ :

Lemma 12: For $\lambda \in\left(\lambda_{1}, \lambda_{2}\right)$ the equation $\Upsilon^{\prime}(u)=0$ has exactly three solutions $0, u_{1},-u_{1}$ in $W_{0}^{1,2}(\Omega)$, where $u_{1} \neq 0$ and $\Upsilon\left(u_{1}\right)=\Upsilon\left(-u_{1}\right)=\inf _{u \in W_{0}^{1,2}(\Omega)} \Upsilon(u)$.

For the Proof, which uses the theory of proper nonlinear Fredholm operators, we refer to [2] $\mathrm{c}$

Now, for the Example to Theorem 3 it is our aim to show that all assumptions (D1) - (D6) are satisfied. For (D1) and (D2) this can be shortly done.

(D1) follows from Lemma 11.

(D2): The set $\left\{u \in W_{0}^{1,2}(\Omega) \mid \Upsilon(u) \leq \alpha\right\}$ is bounded since (19) implies $\Upsilon(u) \rightarrow+\infty$ as $\|u\| \rightarrow \infty$. Moreover, since $\Upsilon$ is continuous and $\alpha>\inf _{u \in W_{0}^{12}(\Omega)} \Upsilon(u)$ we have $M \neq \emptyset$. The remainding part follows from Lemma 12 and from (20).

To verify (D3) we first state

Lemma 13: Under the assumptions stated above it holds inf $\mathrm{u}_{u \in M}\left\|\mathrm{I}^{\prime}(u)\right\|>0$.

Proof: We give a proof by contradiction. If $\inf _{u \in M}\left\|\Upsilon^{\prime}(u)\right\|=0$, then there exists a sequence $\left(u_{m}\right) \subset M$ (i. e. , $\Upsilon\left(u_{m}\right)=\alpha$ for all $m \in N$ ) such that $\left\|\Upsilon^{\prime}\left(u_{m}\right)\right\| \rightarrow 0$ as $m \rightarrow \infty$. The functional $\Upsilon$ satisfies (PS) since it is a functional of the type considered in the Erample to Theorem 1 (denoted there by $\Phi$ ), especially the assumptions of Lemms 2 are satisfied. Hence there is a subsequence $\left(u_{m^{\prime}}\right) \subseteq\left(u_{m}\right)$ such that $u_{m} \rightarrow w$ in $W_{0}^{1,2}(\Omega)$ as $m^{\prime} \rightarrow \infty$. The derivative $\Upsilon^{\prime}$ is continuous, therefore, $\Upsilon^{\prime}(w)=0$. From Lemma 12 there follows either $\Upsilon(w)=\Upsilon(0)>\alpha$ or $\Upsilon(w)=\inf _{u \in W_{0}^{1,2}(n)}<\alpha$, i. e. , $\Upsilon(w) \neq \alpha$. On the other hand, because of the continuity of $\Upsilon$ it holds $\Upsilon(w)=\alpha$, which is a contradiction $\square$

(D3): We set $\beta=\inf _{u \in M}\left\|\Upsilon^{\prime}(u)\right\|$. For each $u \in M$ we choose $\nu_{u} \in W_{0}^{1,2}(\Omega),\left\|\nu_{u}\right\|=1$, such that $\left(\Upsilon^{\prime}(u), \nu_{u}\right)>2 \beta / 3$. Since $\Upsilon^{\prime}$ is continuous, for each $u \in M$ there is an open neighbourhood $N_{u}$ of $u$ in $M$ such that $\left\langle\Upsilon^{\prime}(w), \nu_{u}\right\rangle>\beta / 2$ for all $w \in N_{u}$. The family $\left\{N_{u}\right\}$ is an open covering of $M$. Since every subset of a Banach space is paracompact, there are a locally finite refinement $\left\{N_{i}\right\}$ of $\left\{N_{u}\right\}$ and corresponding $\nu_{i} \in W_{0}^{1,2}(\Omega),\left\|\nu_{i}\right\|=1$, such that $\left\langle\Upsilon^{\prime}(w), \nu_{i}\right\rangle>\beta / 2$ for all $w \in N_{i}, i \in \mathbf{N}$.

Now, let $\left\{\eta_{i}\right\}$ be a partition of unity on $M$ subordinate to $\left\{N_{i}\right\}$, and set $\mu(u)=\sum_{i} \eta_{i}(u) v_{i}$, $u \in M$. Then the mapping $\mu: M \rightarrow W_{0}^{1,2}(\Omega)$ is continuous, $\|\mu(u)\| \leq 1$ for all $u \in M$, and we have $\left\langle\Upsilon^{\prime}(u), \mu(u)\right\rangle=\sum_{i} \eta_{i}(u)\left\langle\Upsilon^{\prime}(u), \nu_{i}\right)>\sum_{i} \eta_{i} \beta / 2=\beta / 2$. Therefore, $\mu(u) \neq 0$ for all $u \in M$. 
We set $\nu(u)=\mu(u) /\|\mu(u)\|$ for $u \in M$. Then, $\nu$ is continuous on $M$ and $\|\nu(u)\|=1$ for all $u \in M$. Furthermore, $\left\langle\Upsilon^{\prime}(u), \nu(u)\right\rangle=\left\langle\Upsilon^{\prime}(u), m(u)\right\rangle /\|m(u)\|>\beta / 2>0$ for all $u \in M$, and (D3) is satisfied.

(D4): $\Upsilon^{\prime}$ is the sum of the uniformly monotone operator $\Upsilon^{\prime}-\Gamma^{\prime}$ and the strongly continuous operator $\Gamma^{\prime}$ (cf. Lemma 11), hence it satisfies (S) $)_{1}$, cf. [10: Chapter 27.1]. Now, we prove that $\Upsilon^{\prime}$ is Lipschitz continuous on $M$ (hence locally Lipschitz continuous). For $u, v \in M, w \in W_{0}^{1,2}(\Omega)$ we get with the Hölder inequality

$$
\begin{aligned}
& \left|\left(\Upsilon^{\prime}(u)-\Upsilon^{\prime}(v), w\right)\right| \\
& \quad \leq\left|\int_{\Omega} \nabla(u-v) \nabla w d x\right|+\lambda\left|\int_{\Omega}(u-v) w d x\right|+\left|\int_{\Omega}(u-v)\left(u^{2}-2 u v+v^{2}\right) w d x\right| \\
& \quad \leq\|u-v\|\|w\|+\lambda\|u-v\|_{2}\|w\|_{2}+\|u-v\|_{4}\left(\left\|u^{2}\right\|_{2}+2\|u v\|_{2}+\left\|v^{2}\right\| \|_{2}\right)\|w\|_{4} .
\end{aligned}
$$

By the Sobolev imbedding $W_{0}^{1,2}(\Omega) \rightarrow L_{4}(\Omega)$ and the fact that the set $M$ is bounded we get $\left|\left\langle\Upsilon^{\prime}(u)-\Upsilon^{\prime}(v), w\right\rangle\right| \leq c\|u-v\|\|w\|$, where the constant $C$ does not depend on $u, v$. Hence $\left\|\Upsilon^{\prime}(u)-\Upsilon^{\prime}(v)\right\| \leq C\|u-v\|$ for all $u, v \in M$, i. e. , $\Upsilon^{\prime}$ is Lipschitz continuous on $M$. Since $M$ is bounded, it immediately follows that $Y$ is bounded on $M$.

(D5): We choose $\bar{M}=\overline{\operatorname{conv} M}$ (the closure of the conver hull of $M$ ). $\bar{M}$ is bounded since $M$ is, and it is well known that $\bar{M}$ is closed with respect to the weak convergence in $W_{0}^{1,2}(\Omega)$.

(D6): $\Phi^{\prime}$ is strongly continuous, by Lemma 11 . It is obvious that $\Phi(u) \geq 0$ on $M$. Furthermore, it holds $\Phi(u)=p^{-1}\|u\|_{p}^{p} \leq C\|u\|^{p}$ because of the Sobolev imbedding $W_{0}^{1,2}(\Omega) \rightarrow L_{p}(\Omega)$ for $p<6$. Thus, $\Phi$ is bounded on $M$, since $M$ is bounded in $W_{0}^{1,2}(\Omega)$. It is easy to see that for $u \in W_{0}^{1,2}(\Omega)$ it holds:

$$
\Phi(u)=0 \Longleftrightarrow u=0 \Longleftrightarrow \Phi^{\prime}(u)=0
$$

So, (D1) - (D6) are fulfilled and we have shown that Theorem 3 applies to our example.

Finally, we will prove that we can choose the number $\sigma$ in the functional $\Psi$ in such a way that the condition $|\Psi(u)|+\left\|\Psi^{\prime}(u)\right\|<\delta$ for all $u \in \bar{M}$ is fulfilled. Note that, by Lemma 11, $\Psi \in C^{1}\left(W_{0}^{1,2}(\Omega), \mathbf{R}\right)$ and $\Psi$ is strongly continuous independent of $\sigma \in \mathbf{R}$.

Lemma 14: For the functional $\Psi$ given above there exists a number $\sigma_{0}>0$ such that for $\sigma$ with $|\sigma|<\sigma_{0}$ it holds $|\Psi(u)|+\left\|\Psi^{\prime}(u)\right\|<\delta$ for all $u \in \bar{M}$.

Proof: a) From (P2) it follows the existence of constants $a_{1}, b_{1} \geq 0$ such that $|P(x, t)| \leq$ $a_{1}+b_{1}|t|^{++1}$ for all $x \in \Omega$. For $u \in W_{0}^{1,2}(\Omega)$ it follows

$$
|\Psi(u)| \leq|\dot{\sigma}| \int_{\Omega}|P(x, u)| d x \leq|\sigma| \int_{\Omega}\left(a_{1}+b_{1}|u|^{\rho+1}\right) d x \leq|\sigma|\left(a_{1}|\Omega|+C b_{1}\|u\|^{\rho+1}\right),
$$


using the imbedding $W_{0}^{1,2}(\Omega) \rightarrow L_{0+1}(\Omega)$ since $s+1<6$.

b) By (P2), for $u, v \in W_{0}^{1,2}(\Omega)$ we have

$$
\begin{aligned}
& \left|\left\langle\Psi^{\prime}(u), v\right\rangle\right| \leq|\sigma| \int_{n}|p(x, u)||v| d x \\
& \leq|\sigma|\left\{\int_{\Omega}|p(x, u)|^{(\bullet+1) / s} d x\right\}^{\bullet /(\bullet+1)}\left\{\int_{\Omega}|v|^{\bullet+1} d x\right\}^{1 /(\bullet+1)} \\
& \leq|\sigma|\left\{\int_{\Omega}\left(a+b|u|^{\bullet}\right)^{(\bullet+1) / \bullet} d x\right\}^{\iota /(\bullet+1)}\left\{\int_{\Omega}|v|^{e+1} d x\right\}^{1 /(\bullet+1)} \\
& \leq|\sigma|\left(a|\Omega|^{a /(a+1)}+b\|u\|_{0+1}^{a}\right)\|v\|_{0+1} \\
& \leq|\sigma|\left(a|\Omega|^{\cdot /(\bullet+1)}+C b\|u\|^{*}\right) C\|v\| \text {. }
\end{aligned}
$$

Since $\bar{M}$ is bounded from a) and b) there follows the existence of a number $\sigma_{0}>0$ such that $|\Psi(u)|<\delta / 2$ and $\left\|\Psi^{\prime}(u)\right\|<\delta / 2$ for all $u \in \bar{M}$.

Remark: The set $M$ consisered in our example is not homeomorphic to the unit sphere in $W_{0}^{1,2}(\Omega)$. To see this, we prove that $M$ consists of at least two connected components. Let $\bar{u}$ be an eigensolution of (17) to the first eigenvalue $\lambda_{1}$, and let $\Sigma=\left\{u \in W_{0}^{1,2}(\Omega):(u, \tilde{u})=0\right\}$ be the hyperplane in $W_{0}^{1,2}(\Omega)$ which is orthogonal to $\bar{u}$. We have, by the variational characterization of the eigenvalues of (17), $\int_{\Omega}|\nabla u|^{2} d x \geq \lambda_{2} \int_{\Omega} u^{2} d x$ for all $u \in \Sigma$. Hence, using (18), for $u \in \Sigma$ it follows

$$
\Upsilon(u)=\frac{1}{2} \int_{\Omega}|\nabla u|^{2} d x-\frac{\lambda}{2} \int_{\Omega} u^{2} d x+\frac{1}{4} \int_{\Omega} u^{4} d x \geq \frac{1}{2}\left(1-\frac{\lambda}{\lambda_{2}}\right) \int_{\Omega}|\nabla u|^{2} d x \geq 0 .
$$

Since $\alpha<0$ it holds $M \cap \Sigma=0$. On the other hand, if $u_{1}$ is the element from Lemma 12, each of the opposite rays $\left\{t u_{1}: t>0\right\}$ and $\left\{t u_{1}: t<0\right\}$ meets $M$ at least in one point, by the continuity of $\Upsilon$ and (20). Hence $M$ has at least two connected components.

\subsection{Functionals on bounded level sets II}

In this subsection we assume:

(E1) $X$ is a real Hilbert space, $\Phi \in C^{1}(X, \mathbf{R})$.

(E2) $M=\{u \in X \mid\|u\|=1\}, \tilde{M}=\{u \in X:\|u\| \leq 1\}$. There exist numbers $k \in \mathrm{N}$, $\rho>0$, and an add mapping $\psi_{0} \in C\left(S^{k-1}, M\right)$ such that $\psi_{0}\left(S^{k-1}\right) \subset M_{\rho}=$ $\{u \in M: \Phi(u)>\rho\}$, where $S^{k-1}$ is the unit sphere in $\mathbf{R}^{k}$.

(E3) $\Phi^{\prime}: X \rightarrow X^{\prime}$ is strongly continuous. $\Phi(u) \geq 0$ for all $u \in X$, and $\Phi$ is bounded on $M$. For $u \in X$ it holds $\Phi(u)=0 \Longleftrightarrow u=0 \Longleftrightarrow \Phi^{\prime}(u)=0$. 
Theorem 4: Under the assumptions (E1) to (E3) the following holds:

1) There is a real number $c>\rho$ such that crit $_{M_{,}} \Phi \neq 0$.

2) For every $\epsilon>0$ there exists $\delta>0$ such that for all $\Psi \in C^{1}(X, \mathbf{R})$ with strongly continuous derivative $\Phi^{\prime}$ and $|\Psi(u)|+\left\|\Psi^{\prime}(u)\right\|<\delta$ for all $u \in \bar{M}$ there is a number $c_{*} \in(c-\epsilon, c+\epsilon)$ for which crit $_{M_{1}, \Phi_{*}} \Phi_{*} \neq \emptyset$ (where $\Phi_{*}=\Phi+\Psi$ ).

Proof: 1) It is our aim to apply Proposition 3, hence we have to verify (H1) to (H4).

(H1) is obviously satisfied.

(H2): We define

$$
\begin{aligned}
& B=\left\{\begin{array}{l|l}
B \subset M_{\rho} & \begin{array}{l}
\text { There is an odd mapping } \psi \in C\left(S^{k-1}, M_{\rho}\right) \\
\text { such that } B=\psi\left(S^{h-1}\right)
\end{array}
\end{array}\right\} . \\
& \mathcal{K}=\left\{\begin{array}{l|l}
K \subset M_{\rho} & \begin{array}{l}
\text { There is a } B \in B \text { and } a \bar{d} \in C\left(B \times[0,1], M_{p}\right) \text { with } \\
\vec{d}(u, 0)=u \text { for all } u \in B \text { such that } K=\bar{d}(B, 1)
\end{array}
\end{array}\right\} .
\end{aligned}
$$

Obviously $\mathcal{B} \subseteq \mathcal{K}$, and since $\psi_{0}\left(S^{k-1}\right) \in B$ according to (E2) it follows $\mathcal{K} \neq \emptyset$. Now for the number $c=\sup _{K \in \mathcal{K}} \inf _{u \in K} \Phi(u)$ it holds $c<+\infty$ since $\Phi$ is bounded on $M$. Furthermore, because $\psi_{0}\left(S^{k-1}\right)$ is compact it holds $\inf _{u \in \psi_{0}\left(S^{k-1}\right)} \Phi(u)>\rho$, hence

$$
c>\rho \text {. }
$$

(H3): We define

$$
\mathcal{D}=\left\{\begin{array}{l|l}
d \in C(M, M) & \begin{array}{l}
\text { There is a } \bar{d} \in C(M \times[0,1], M) \text { such that } \bar{d}(\cdot, t) \text { is a homeo- } \\
\text { morphiom of } M \text { onto itself for all } t \in[0,1], \bar{d}(u, 0)=u \text { and } \\
\Phi(\bar{d}(u, t)) \geq \Phi(u) \text { for all }(u, t) \in M \times[0,1] \text { and } d(u)=\bar{d}(u, 1) \\
\text { for all } u \in M
\end{array}
\end{array} .\right.
$$

To prove that $\mathcal{K}$ is invariant under $\mathcal{D}$ let $K \in \mathcal{K}$, i. e. , $K=\bar{d}_{1}(B, 1)$, where $B$ and $\bar{d}_{1}$ are according to (21). Furthermore, let $d_{2} \in \mathcal{D}, d_{2}(\cdot)=\bar{d}_{2}(\cdot, 1)$. If we set

$$
\bar{d}(u, t)= \begin{cases}\bar{d}_{1}(u, 2 t) & \text { for } u \in B, t \in[0,1 / 2] \\ \bar{d}_{2}\left(\bar{d}_{1}(u, 1), 2 t-1\right) & \text { for } u \in B, t \in(1 / 2,1],\end{cases}
$$

then $d_{2}(K)=\bar{d}(B, 1)$, and it is easy to see that $\bar{d} \in C\left(B \times[0,1], M_{\rho}\right)$. Hence $d_{2}(K) \in \mathcal{K}$.

(H4): It is our aim to apply Lemma 4. If we set $\Upsilon(u)=\|u\|^{2}, u \in X$, and $\alpha=1$, then it is immediately clear that (C1), (C2), and (C4) are fulfilled, where $\left\langle\Upsilon^{\prime}(u), v\right\rangle=2(u, v)$ for $u, v \in X$. Furthermore, in (C3) we can choose $\nu(u)=u, u \in M$. Assumption (C5) holds, since $\bar{M}=\{u \in X:\|u\|=1\}$ is bounded and closed with respect to the weak convergence in $X$.

For (H4) it remains to show that $\Phi$ satisfies (PS) $c$ on $M$. For this, we use Lemma 5. From 
(E3) there follow assumptions $c$ ) and d) of this lemma for all $c \neq 0$. To verify b), let $\left(u_{m}\right) \subset X$ such that $u_{m} \rightarrow u$ in $X, \Upsilon^{\prime}\left(u_{m}\right) \rightarrow v$ in $X^{\prime}$ as $m \rightarrow \infty$. It holds $\Upsilon^{\prime}\left(u_{m}\right) \rightarrow \Upsilon^{\prime}(u)$ as $m \rightarrow \infty$ since $\left\langle\Upsilon^{\prime}\left(u_{m}\right), w\right\rangle=2\left(u_{m}, w\right),\left\langle\Upsilon^{\prime}(u), w\right\rangle=2(u, w)$ for all $w \in X$. Hence $v=\Upsilon^{\prime}(u)$. The estimate

$$
\begin{aligned}
2\left\|u_{m}-u\right\|^{2} & =2\left(u_{m}-u, u_{m}-u\right)=\left\langle\Upsilon^{\prime}\left(u_{m}\right)-\Upsilon(u), u_{m}-u\right\rangle \\
& \leq\left\|\Upsilon^{\prime}\left(u_{m}\right)-\Upsilon^{\prime}(u)\right\|\left\|u_{m}-u\right\|
\end{aligned}
$$

yields $u_{m} \rightarrow u$ in $X$ as $m \rightarrow \infty$.

2) Let $\epsilon>0$ be given. Set

$$
\tau=\inf \left\{\left\|\Phi^{\prime}(u)\right\| \mid u \in \bar{M}, \Phi(u) \in(c / 2,3 c / 2)\right\} .
$$

The same proof as in Lemma 9 yields $\tau>0$. Now, we choose positive numbers $\sigma, \delta$ such that

$$
\sigma<\min \{\epsilon / 2, c-\rho\}, \delta<\min \{\epsilon / 2, \tau\}, \sigma+2 \delta<\min \{c / 2, c-\rho\} \text {. }
$$

Note that $c>\rho$, by (22). We shall apply Propositions 4 and 6, so we have to verify (H5) to (H9).

(H5): Let $\delta$ be the number choosen accordingly to (25). Under the assumptions of part 2) of our theorem (H5) is fulfilled.

(H6),(H7): For the construction of a class $\mathcal{K}_{*}$, let $\sigma$ be taken accordingly to (25) and choose a set $K_{\sigma} \in \mathcal{K}$ such that $\inf _{u \in K_{\sigma}} \Phi(u)>c-\sigma$. We define

$D_{*}=\left\{\begin{array}{l|l}d \in C(M, M) & \begin{array}{l}\text { There is a } \bar{d} \in C(M \times[0,1], M) \text { such that } \bar{d}(\cdot, t) \text { is a homeo- } \\ \text { morphism of } M \text { onto itself for all } t \in[0,1], \bar{d}(u, 0)=u \text { and } \\ \Phi_{*}(\bar{d}(u, t)) \geq \Phi_{*}(u) \text { for all }(u, t) \in M \times[0,1], \text { and } d(u)=\bar{d}(u, 1) \\ \text { for all } u \in M\end{array}\end{array}\right\}$

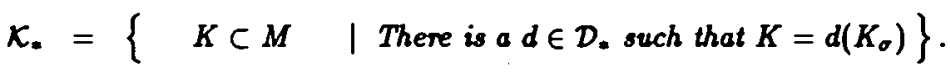

It holds $\mathcal{K}_{*} \neq 0$ since $K_{\sigma}=i d\left(K_{\sigma}\right) \in \mathcal{K}_{*}^{\prime}$, where id is the identity mapping on $M$. It remains to show $\mathcal{K}_{*} \subseteq \mathcal{K}$. Let $K \in \mathcal{K}_{*}$, i. e. , $K=d_{3}\left(K_{\sigma}\right), d_{2} \in \mathcal{D}_{*}, d_{2}(\cdot)=\bar{d}_{2}(\cdot, 1)$. Since $K_{\sigma} \in \mathcal{K}$, there is a $B \in B$ and a $\bar{d}_{1} \in C\left(B \times[0,1], M_{\rho}\right)$ with $\bar{d}_{1}(u, 0)=u$ for all $u \in B$ such that $K_{\sigma}=\bar{d}_{1}(B, 1)$. If we construct a mapping $\bar{d} \in C(B \times[0,1], M)$ accordingly to (23) we have $K=\bar{d}(B, 1)$. Thus, we have to show $\bar{d} \in C\left(B \times[0,1], M_{\rho}\right)$, which is proven if we can show

$$
\Phi(\bar{d}(u, t))>\rho
$$

for all $u \in B, t \in[0,1]$. Obviously (26) holds for $t \in[0,1 / 2]$ by construction of $\bar{d}$. To verify inequality (26) for all $t$ it suffices to prove

$$
\Phi\left(\bar{d}_{2}(u), t\right)>\rho
$$


for all $u \in K_{\sigma}, t \in[0,1]$, by (23) and $K_{\sigma}=\bar{d}_{1}(B, 1)$. For $u \in K_{\sigma}$ it holds $\Phi(u)>\rho+2 \delta$ (note (25)), hence $\Phi_{*}(u)>\rho+\delta$ since $|\Psi(u)|<\delta$. From the definition of $\mathcal{D}_{*}$ it follows $\Phi_{*}\left(\bar{d}_{2}(u, t)\right) \geq$ $\Phi_{*}(u)>\rho+\delta$, therefore, $\Phi\left(\bar{d}_{2}(u, t)\right)>\rho$ for all $t \in[0,1]$, and (27) is proven.

(H8): $\mathcal{D}_{*}$ is a semigroup with respect to the composition of mappings. This immediately becomes clear if we use a construction analogous to (23). Now, by construction, $\mathcal{K}_{*}$ is invariant under $\mathcal{D}$.

(H9): If we choose $\tau$ accordingly to (24), then the verification of (H4) in part 1) of our proof shows that all assumptions of Lemma 6 are fulfilled. Hence the functional $\Phi$ satisfies (PS) for all $c \in(c / 2+\delta, 3 c / 2-\delta)$. Proposition 4 yields $c_{*} \in(c-\delta-\sigma, c+\delta]$, and, by (25), it holds $c / 2+\delta<c-\delta-\sigma, 3 c / 2-\delta>c+\delta$. Therefore, $\Phi_{*}$ satisfies (PS) $)_{c_{\oplus}}$, and from Lemma 4 it follows that (H9) is fulfilled.

Now, Proposition 4 shows crit $M_{\mu_{*}} \Phi_{*} \neq \emptyset$, and $c_{*} \in(c-\epsilon, c+\epsilon)$, because inequalities (25) field $c_{*} \in(c-\delta-\sigma, c+\delta] \subset(c-\epsilon, c+\epsilon) \mathbf{u}$

Remarks: 1) It is not possible to choose $\mathcal{K} *=\mathcal{K}$ because $\mathcal{K}$ is not invariant under $\mathcal{D}_{\text {.. If }}$ $K \in \mathcal{K}, d \in \mathcal{D}_{*}$, then in general $d(K)$ is not a subset of $M_{\rho}$. 2) In [4] there are used classes $\mathcal{K}$ of the type considered above to obtain a series of distinct critical values by varying $k$.

Example to Theorem 4: Let $\Omega \subset \mathbf{R}^{n}$ and $X=W_{0}^{1,2}(\Omega)$ as in the Example to Theorem 1. We consider the functionals $\Phi$ and $\Psi$ from the Example to Theorem 3 under the assumption $1<q<+\infty$ if $n \leq 2$ and $1<q<2 n /(n-1)$ if $n>2$. It is our aim to prove that all assumptions of Theorem 4 for our example are fulfilled.

(E1),(E3): They follows from Lemms 11. To see that $\Phi^{\prime}(u)=0$ implies $u=0$ we use the identity $\left\langle\Phi^{\prime}(u), u\right\rangle=q \Phi(u)$ for all $u \in W_{0}^{1,2}(\Omega)$.

(E2): Let $k \in N$ be an arbitrary number. Furthermore, let $\left(u_{m}\right)$ be an orthonormized basis in $W_{0}^{1,2}(\Omega)$. Set $E_{k}=\operatorname{span}\left\{u_{1}, \ldots, u_{k}\right\}$, and let $\psi_{0}: \mathbf{R}^{k} \rightarrow E_{k}$ be the canonical isomorphism. Since the set $\psi_{0}\left(S^{k-1}\right)$ (where $S^{k-1} \subset \mathbf{R}^{k}$ ) is compact and since $\Phi(u)>0$ for all $u \in M$ it follows $\inf _{u \in \psi_{0}\left(S^{k-1}\right)} \Phi(u)>0$. Now, for every number $\rho$ satisfying $0<\rho<\inf _{u \in \psi_{0}\left(a^{k-1}\right)} \Phi(u)$ assumption (E2) is fulfilled.

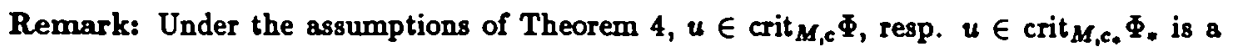
weak solution of the eigenvalue problem

resp.

$$
-\mu \Delta u=u|u|^{q-2} \quad \text { in } \Omega, u=0 \text { on } 8 \Omega \text {. }
$$

$$
-\mu \Delta u=u|u|^{q-3}+\sigma p(x, u) \quad \text { in } \Omega, u=0 \text { on } \partial \Omega \text {. }
$$

Finally, we remark that the assertions of Lemma 11 for $\Phi$ and $\Phi$ are valid and that Lemma 14 is also true under the setting of this example. 


\section{References}

[1] BAhri,A. and H.Berestycky: A Perturbation Method in Critical Point Theory and Applications. Trans. Amer. Math. Soc. 267 (1981), 1-32.

[2] Berger,M.S.: Nonlinear Problems with Exactly Three Solutions. Indians Univ. Math. J. 28 (1979), 689698.

[3] De Fiougmedo,D.G.: Lectures on the Ekeland Variational Principle and Detours. Lecture Note. Trieste: International Centre for Theoretical Physics 1988.

[4] Krasnosel'skir.M.A.: Topological Methods in the Theory of Nonlinear Integral Equations. Orford London - New York - Paris. Pergamon Press 1964.

[5] Rusinowitz,P.H.: Minimax Methods in Critical Point Theory with Applications to Differential Equations. CBMS Regional Conf. Ser. in Math., no. 65. Providence: Amer. Math. Soc. 1986.

[6] Reeken,M.: Stability of Critical Points under Small Perturbations I and II. Manuscripta Math. 7 (1972), 387-411 and 8 (1973), 69-92.

[7] Struwe,M.: Infinitely Many Critical Points for Punctionals which are Not Even and Applications to Superlinear Boundary Value Problems. Manuscripta Math. 32 (1980), 335-364.

[8] ZeLDLEn, E.: The Ljusternik-Schnirelman Theory for Indefinite and not Necessarily Odd Nonlinear Operators and its Applications. Nonlinear Anal. 4 (1980), 451-489.

[9] ZeIdLer,E.: Ljusternik-Schnirelman Theory on General Level Sets. Math. Nachr. 129 (1986), 235-259.

[10] Zerder, E.: Nonlinear Functional Analysis and its Applications, Vols. I - IV. New York - Berlin Heidelberg - Tokyo. Springer-Verlag 1985-1990.

Received 02.10.1990, in revised form 15.09.1991.

Dr. Frank Benkert

Institut für Mathematik der Universität Leipzig

Augustusplatz 10

D (Ost) - 7010 Leipzig 